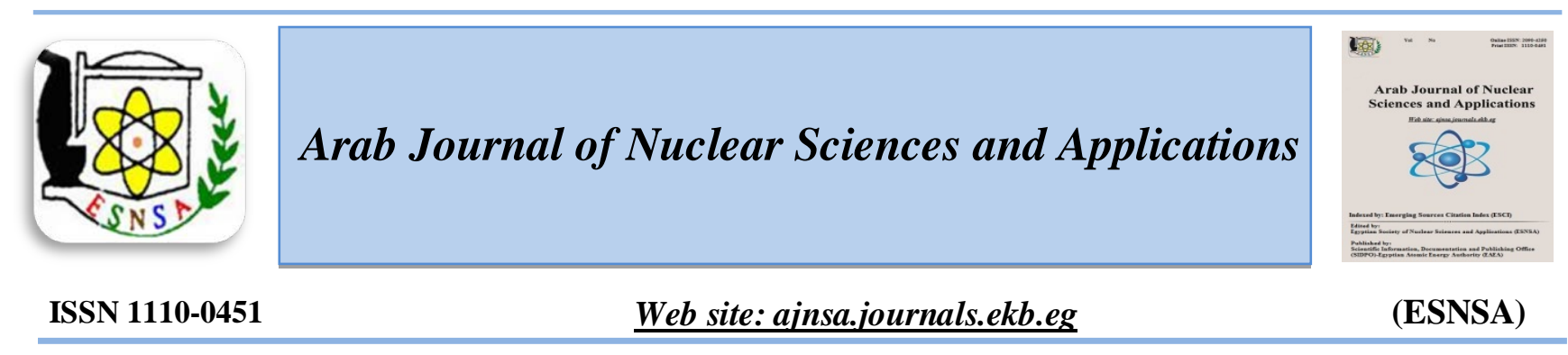

\title{
Production of a Charged Higgs Boson with Chargino and Neutralino
}

\author{
T.A. El-Azim \\ Physics Department, Faculty of Science, Cairo University, Giza, Egypt
}

\begin{abstract}
Received $27^{\text {th }}$ Nov. 2017 The reaction $e^{-}+e^{+}\left(p_{2}\right) \rightarrow H^{-}+\widetilde{\chi}_{1}^{+}+\widetilde{\chi}_{1}^{o}$ in MSSM model is studied. The production total cross Accepted $27^{\text {th }}$ Dec. 2017 section is evaluated in specifics by numerical integration over the kinematically allowed range. Three values for the parameter $\tan \beta(5,15,35)$ have been used to study the production process with a proposal mass parameter $M(150,300 \mathrm{GeV})$. It was found that the dominant production can reach about $10^{-4} \mathrm{pb}$ in the allowed range of parameters. The maximum cross section achieved reaches the value $1.6853 \times 10^{-5} \mathrm{pb}$ for $\tan \beta=5$ and $M 2=300 \mathrm{GeV}$ at a center of mass energy, $E_{C M}=880 \mathrm{GeV}$ for the diagram no. 2 which proceeds through the photon propagator, $\gamma$. A maximum of $1.0836 \times 10^{-4}$ pb was obtained for $\tan \beta=15$ and $M 2=300 \mathrm{GeV}$ at a center of mass energy, $E_{C M}=860 \mathrm{GeV}$ for the diagram no. 28 which proceeds through the exchange of the scalar neutrino propagator, $\tilde{v}_{e}$. For $\tan \beta=35$ and $M 2=300 \mathrm{GeV}$ at a center of mass energy, $E_{C M}=900 \mathrm{GeV}$, the cross section obtained, reached the value $1.8623 \times 10^{-4} \mathrm{pb}$.
\end{abstract}

Keywords: MSSM model/ Higgs Boson/ Neutralino/ Charged Higgs.

\section{Introduction}

The phenomenological predictions of supersymmetry (SUSY) may be divided into three categories [1, 2, 3]:

1. Reflections of super symmetric lagrangian in the Standard Model (SM) $[4,5,6,7]$ phenomenology, including relations among the gauge coupling constants from supersymmetric ${ }^{8,9,10}$ grand unification and the presence of a heavy top quark and light Higgs scalar;

2. The prediction of new particles with the correct spin and quantum numbers assignments to be super partners of the standard model particles; and

3. Well-defined quantitative relations among the couplings and masses of these new particles.

While the predictions of (1) are of great interest, their verification is clearly no substitute for direct evidence. The discovery of a large number of particles in category (2) would be a strong support for supersymmetry. On the other hand, the most compelling confirmation of supersymmetry would likely be the precise verification of the relations of category (3). This would be especially true if, initially, only a small set of candidate supersymmetric partners are observed.

Most discussions of supersymmetry at future high energy colliders have concentrated on the question of particle searches. From one point of view, this is reasonable, because the existence of supersymmetric partners is unproven and this is a prerequisite for any further analysis. On the other hand, the discovery of the first evidence for supersymmetry, or for any other theoretical extension of the standard model, will begin a program of detailed experimental investigation of the new sector of particles required by this extension.

Supersymmetry provides a particularly interesting subject for studies of the detailed analysis of

Corresponding author: taztabaz@sci.cu.edu.eg

DOI: 10.21608/ajnsa.2018.2177.1016

(C) Scientific Information, Documentation and Publishing Office (SIDPO)-EAEA 
physics beyond the standard model. Super symmetry are weakly coupled, and so their consequences can be worked out straightforward using perturbative computations. At the same time, supersymmetric models depend on a large number of unknown parameters, and different choices for these parameters yield qualitatively different realizations of possible new physics. Thus the phenomenology of supersymmetry is quite complex. Eventually, if supersymmetry does give a correct model of nature, the colliders of the next generation will be expected to determine the supersymmetric parameters, and their values will become clues that take us a step closer to a fundamental theory.

In the minimal supersymmetric extension of the standard model, MSSM, among the lightest supersymmetric particles there are four neutralinos (the super symmetric partners of the neural electroweak gauge and Higgs bosons). In most scenarios, apart from the lightest supersymmetric particle (LSP), which is in general assumed to be the lightest neutralino $\left(\tilde{\chi}_{1}^{o}\right)$ (stable and invisible), the particles that could be first observed at future colliders are the next-to-lightest neutralino $\left(\tilde{\chi}_{2}^{o}\right)$ and the light chargino $\left(\tilde{\chi}_{1}^{ \pm}\right)^{11,12,13}$. Therefore, any reasonable large supersymmetric signal must involve either the second lightest neutralino $\left(\tilde{\chi}_{2}^{o}\right)$ or the lighter charginos $\left(\tilde{\chi}_{1}^{ \pm}\right)$. In general, we cannot assume that the second lightest neutralino is heavier than the lighter chargino, since, $m_{\widetilde{\chi}_{2}^{0}}{ }^{14}$ is not independent of $m_{\widetilde{\chi}_{1}^{o}}$ and $m_{\widetilde{\chi}_{1}^{ \pm}}$. In fact, in the region of parameter space in which charginos production is accessible to the future $e^{-} e^{+}$ colliders, $\tilde{\chi}_{2}^{o}$ and $m_{\widetilde{\chi}_{1}^{ \pm}}$are very roughly degenerate, with the mass difference typically in the range $-10 \mathrm{GeV} \leq m_{\tilde{\chi}_{2}^{o}}-m_{\widetilde{\chi}_{1}^{ \pm}} \leq 20 \mathrm{GeV}$.

When $m_{\widetilde{\chi}_{2}^{o}}<m_{\tilde{\chi}_{1}^{ \pm}}$, it is possible for the lighter chargino to decay through cascade decays to a $\tilde{\chi}_{2}^{o}$, which in turn decays to an LSP.
The $e^{-} e^{+}$colliders have been playing complementary roles to the hadron colliders in supersymmetry searches. In general, $e^{-} e^{+}$ colliders have reasonable signal rates in a clean environment with a definite center-of-mass energy, enabling us to perform precision measurements of particles' masses, lifetimes, and various different cross-sections, while hadron colliders provide opportunities to quickly survey high energy frontier. In particular, the production of $\tilde{\chi}_{1}^{o} \tilde{\chi}_{2}^{o}$ pairs at $e^{-} e^{+}$colliders could allow the study of a wide region of the supersymmetry parameter space.

Owing to the relatively large cross-section of two body final state reactions, they can be used to search for supersymmetric particles with masses up to the beam energy. In this study, the production of certain three body final state reactions are calculated to improve the sensitivity in searching for supersymmetric particles.

\section{The production of a charged higgs boson}

The total cross section for the process $e^{-}\left(p_{1}\right) e^{+}\left(p_{2}\right) \rightarrow H^{-}\left(p_{3}\right) \tilde{\chi}_{1}^{+}\left(p_{4}\right) \tilde{\chi}_{1}^{o}\left(p_{5}\right)$ for different topologies and propagators are calculated and represented graphically. There are 28 different Feynman diagrams (tree level approximation) for which we gave the matrix element corresponding to each diagram these are printed in figure 1 . Diagrams with the same topology that can be obtained by changing the indices are represented once in these figures.

The following is the set of Feynman diagrams which were used to calculate the cross section of the associated production of a charged Higgs boson with a chargino and a neutralino. Our momentum notation $e^{-}\left(p_{1}\right), e^{+}\left(p_{2}\right), H^{-}\left(p_{3}\right)$, $\tilde{\chi}_{1}^{+}\left(p_{4}\right)$ and $\tilde{\chi}_{1}^{o}\left(p_{5}\right)$ :

\section{Matrix elements}

The following is the set of matrix elements corresponding to the Feynman diagrams in figures 1 used in our calculations:

$$
\begin{gathered}
M_{1}=\bar{v}\left(p_{2}\right) A \gamma_{\mu} u\left(p_{1}\right) P_{\gamma}^{\mu v}\left(p_{1}+p_{2}\right) J\left(p_{3}+p_{4}-p_{5}\right)_{v} D_{H^{+}}\left(p_{3}+p_{4}\right) \bar{u}\left(p_{4}\right)\left(Q_{i j}^{L} P_{L}+Q_{i j}^{R} P_{R}\right) v\left(p_{3}\right) \\
M_{2}=\bar{v}\left(p_{2}\right) A \gamma_{\mu} u\left(p_{1}\right) P_{\gamma}^{\mu v}\left(p_{1}+p_{2}\right) \bar{u}\left(p_{4}\right) V \gamma_{\mu} D_{\widetilde{v}_{1}^{+}}\left(p_{3}+p_{4}\right)\left(\boldsymbol{p}_{3}+\boldsymbol{p}_{4}+m_{\widetilde{\chi}_{1}^{+}}\right)\left(Q_{i j}^{L} P_{L}+Q_{i j}^{R} P_{R}\right) v\left(p_{3}\right) \\
M_{3}=\bar{v}\left(p_{2}\right) \gamma_{\mu}\left(B^{L} P_{L}+B^{R} P_{R}\right) u\left(p_{1}\right) P_{Z}^{\mu v}\left(p_{1}+p_{2}\right) H\left(p_{1}+p_{2}\right) D_{H^{+}}\left(p_{3}+p_{4}\right) \bar{u}\left(p_{4}\right)\left(Q_{i j}^{L} P_{L}+Q_{i j}^{R} P_{R}\right) v\left(p_{3}\right) \\
M_{4,5}=\bar{v}\left(p_{2}\right) \gamma_{\mu}\left(B^{L} P_{L}+B^{R} P_{R}\right) u\left(p_{1}\right) P_{Z}^{\mu \nu}\left(p_{1}+p_{2}\right) \bar{u}\left(p_{4}\right) \gamma_{\mu}\left(W_{i j}^{L} P_{L}+W_{i j}^{R} P_{R}\right) D_{\widetilde{\chi}_{1}^{+}}\left(p_{3}+p_{4}\right) \\
\left(\boldsymbol{p}_{3}+\boldsymbol{p}_{4}+m_{\widetilde{\chi}_{k}^{+}}\right)\left(Q_{i j}^{L} P_{L}+Q_{i j}^{R} P_{R}\right) v\left(p_{3}\right)
\end{gathered}
$$


where $\mathrm{k}=1,2$

$M_{6,7,8,9}=\bar{v}\left(p_{2}\right) \gamma_{\mu}\left(B^{L} P_{L}+B^{R} P_{R}\right) u\left(p_{1}\right) P_{Z}^{\mu v}\left(p_{1}+p_{2}\right) v\left(p_{3}\right) \gamma_{\mu}\left(S_{i j}+S_{i j}^{\prime} \gamma_{5}\right) D_{\widetilde{\chi}_{k}^{o}}\left(p_{4}+p_{5}\right)$

$$
\left(\boldsymbol{p}_{\mathbf{4}}+\boldsymbol{p}_{\mathbf{5}}+m_{\widetilde{\chi}_{k}^{o}}\right)\left(Q_{i j}^{L} P_{L}+Q_{i j}^{R} P_{R}\right) \bar{u}\left(p_{4}\right)
$$

where $\mathrm{k}=1,2,3,4$

$$
\begin{aligned}
& M_{10,16,17,18,19,24,25,26}=\bar{v}\left(p_{2}\right)\left(N_{i j}+N_{i j}^{\prime} \gamma_{5}\right) v\left(p_{3}\right) D_{\tilde{e}_{h}}\left(p_{1}-p_{3}\right) \bar{u}\left(p_{4}\right)\left(Q_{i j}^{L} P_{L}+Q_{i j}^{R} P_{R}\right) \\
& D_{\widetilde{\chi}_{k}^{o}}\left(p_{3}+p_{5}\right)\left(\boldsymbol{p}_{\mathbf{3}}+\boldsymbol{p}_{\mathbf{5}}+m_{\widetilde{\chi}_{k}^{o}}\right)\left(N_{h j}+N_{h j}^{\prime} \gamma_{5}\right) u\left(p_{1}\right) \\
& M_{11,12,13,14,20,21,22,23}=\bar{v}\left(p_{2}\right)\left(N_{h j}+N_{h j}^{\prime} \gamma_{5}\right)\left(\boldsymbol{p}_{\mathbf{4}}+\boldsymbol{p}_{5}+m_{\widetilde{\chi}_{k}^{o}}\right) D_{\widetilde{\chi}_{k}^{o}}\left(p_{4}+p_{5}\right) \bar{u}\left(p_{4}\right) \\
& \text { where } \mathrm{k}=1,2,3,4 \text { and } \mathrm{h}=\mathrm{L}, \mathrm{R} \text {. } \\
& \left(Q_{i j}^{L} P_{L}+Q_{i j}^{R} P_{R}\right) D_{\tilde{e}_{h}}\left(p_{1}-p_{3}\right) v\left(p_{3}\right)\left(N_{h j}+N_{h j}^{\prime} \gamma_{5}\right) u\left(p_{1}\right)
\end{aligned}
$$

$M_{27,28}=\bar{v}\left(p_{2}\right)\left(T_{i}^{L} P_{L}+T_{i}^{R} P_{R}\right) v\left(p_{3}\right) D_{\tilde{e}_{R}}\left(p_{2}-p_{3}\right) \bar{u}\left(p_{4}\right)\left(Q_{i j}^{L} P_{L}+Q_{i j}^{R} P_{R}\right)\left(\boldsymbol{p}_{\mathbf{4}}+\boldsymbol{p}_{5}+m_{\tilde{\chi}_{k}^{+}}\right)$

$$
D_{\widetilde{\chi}_{k}^{+}}\left(p_{4}+p_{5}\right)\left(T_{i}^{L} P_{L}+T_{i}^{R} P_{R}\right) u\left(p_{1}\right)
$$

where $\mathrm{k}=1,2$

$M_{15}=\bar{v}\left(p_{2}\right) T_{i}^{R} P_{R} v\left(p_{3}\right) D_{\tilde{e}_{L}}\left(p_{2}-p_{3}\right) L\left(p_{1}+p_{2}-p_{4}-p_{3}\right) D_{\tilde{v}_{e}}\left(p_{1}-p_{4}\right) \bar{u}\left(p_{4}\right)$

and

$$
\left(T_{i}^{L} P_{L}+T_{i}^{R} P_{R}\right) u\left(p_{1}\right)
$$

$$
D_{x}(q)=\frac{1}{\left(q^{2}-m_{x}^{2}\right)}, P_{Z, \gamma}^{\mu \nu}=\frac{-g^{\mu \nu}+\frac{g^{\mu} g^{v}}{m_{x}^{2}}}{q^{2}-m_{x}^{2}+i m_{Z, \gamma} \Gamma_{Z, \gamma}}
$$
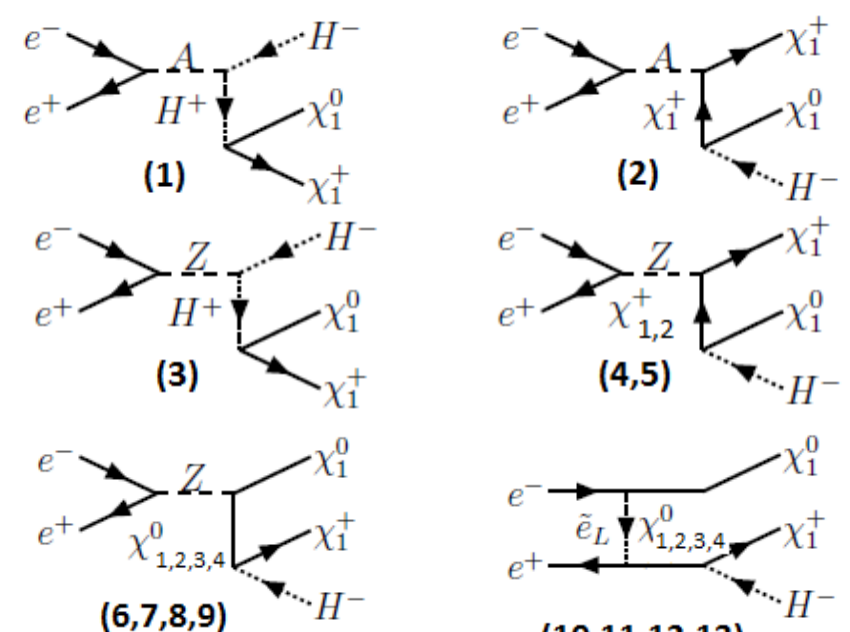

$(10,11,12,13)$
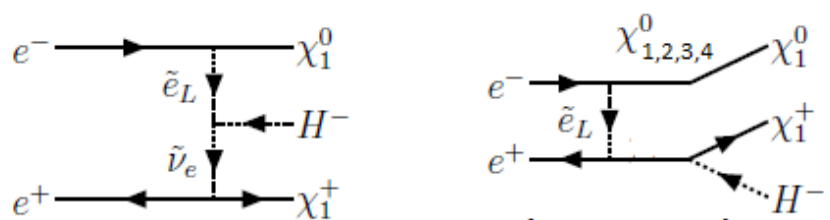

(14)
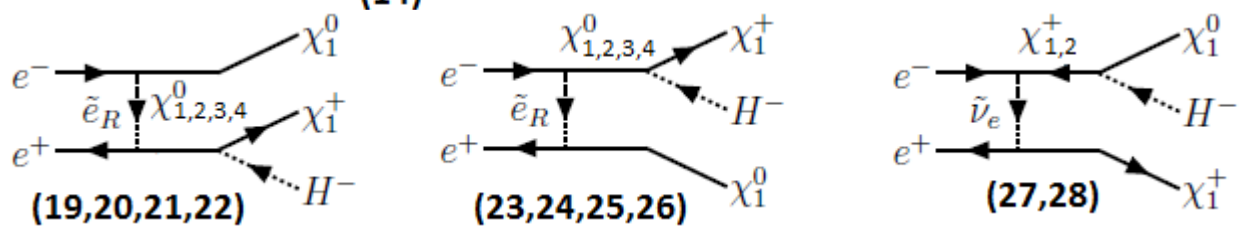

Fig. (1): Feynman diagrams for the reaction: $e^{-}\left(p_{1}\right) e^{+}\left(p_{2}\right) \rightarrow H^{-}\left(p_{3}\right) \tilde{\chi}_{1}^{+}\left(p_{4}\right) \tilde{\chi}_{1}^{o}\left(p_{5}\right)$ 


\section{Cross sections}

In order to calculate the differential cross sections, and hence, the total cross section, we need first to obtain the squared matrix element for each Feynman diagram, the use of the trace theorems was made. Later, an average over the initial spin polarizations of the electron and positron pair and the sum over the final spin states of the outgoing particles arising from each initial spin state is carried out. The total cross section as a function of the center of mass energy is then calculated.

Calculations [13, 25, 27] were done for the following cases: $\tan \beta=05, \tan \beta=15$ and $\tan \beta$ = 35 where $M 2=150$ or $M 2=300$ for each case of $\tan \beta$. All the results are given, but we choose, as examples of our calculations, the first case, the lowest case, the higher tow cases and finally the total cross section in the following figures.
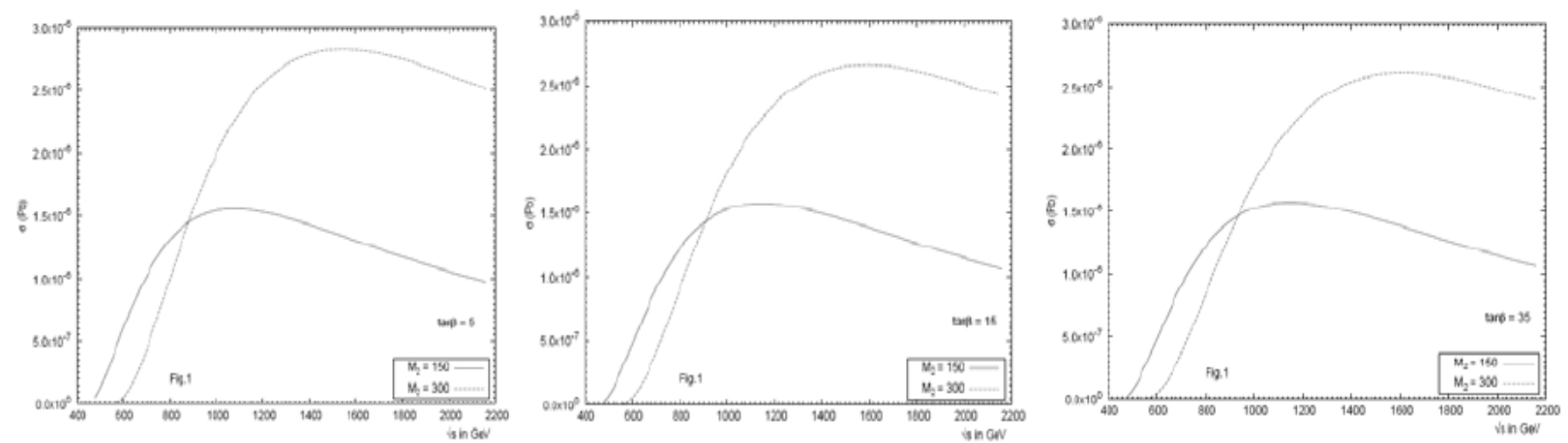

Fig. (2): Cross sections for diagram no. 1 in figure 1
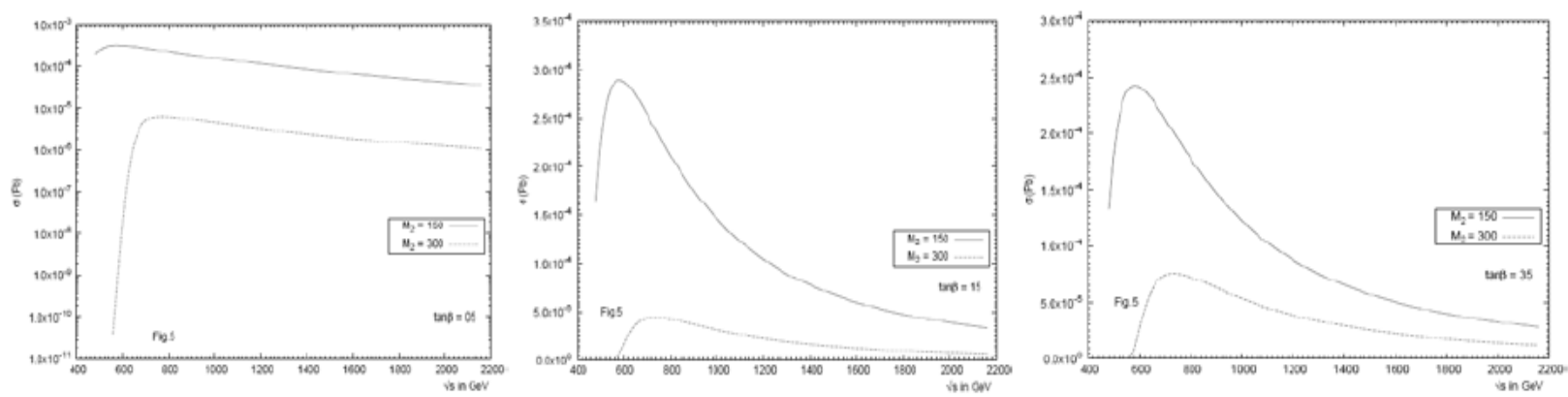

Fig. (3): Cross sections for diagram no. 5 in figure 1 (the lowest)
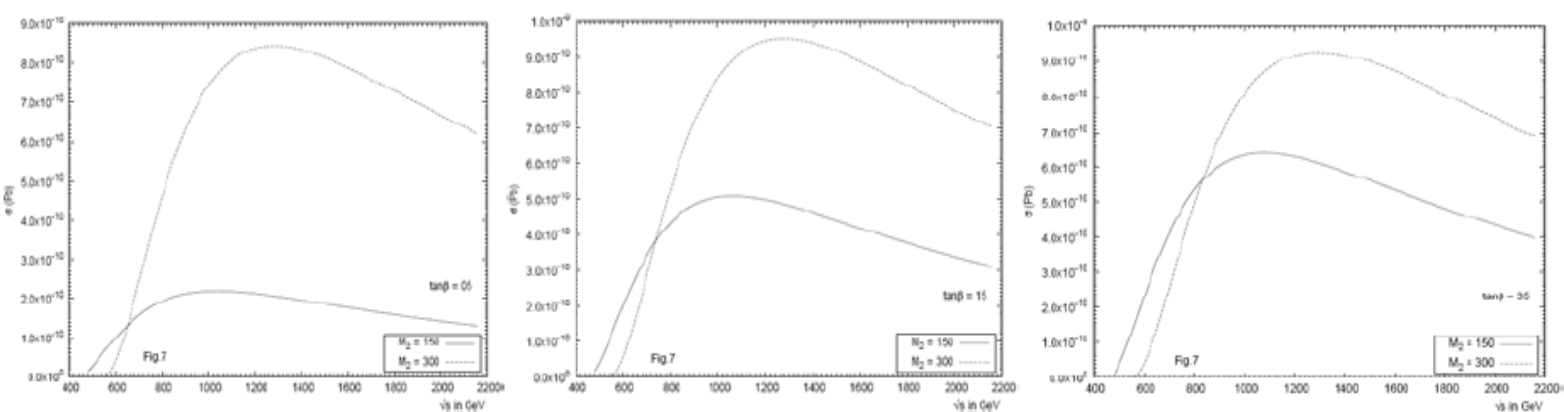

Fig. (4): Cross sections for diagram no. 7 in figure 1 (the $1^{\text {st }}$ highest) 

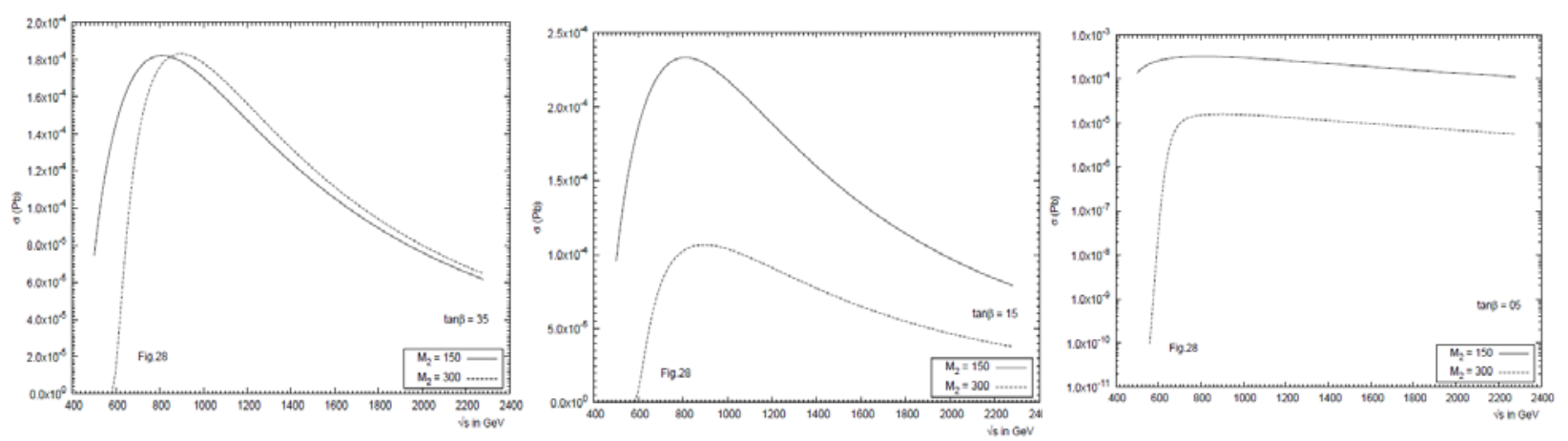

Fig. (5): Cross sections for diagram no. 28 in figure 1 (the $2^{\text {nd }}$ highest)
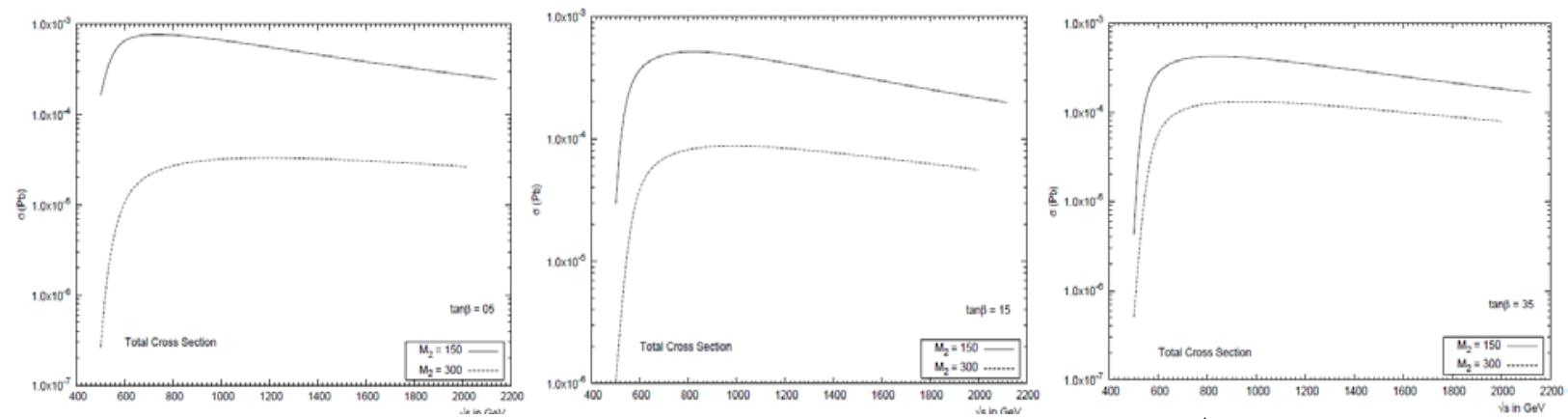

Fig. (6): Total cross section for the reaction $e^{-} e^{+} \rightarrow H^{-} \widetilde{\chi}_{1}^{+} \widetilde{\chi}_{1}^{o}$

\section{Conclusion}

Results of the previous section are summarized in table 1 for $M 2=150 \mathrm{GeV}$, and table 2 for $M 2=$ $300 \mathrm{GeV}$. From these results, it is clear that the reaction is most probably to proceed through diagram no. 5 through the exchange of a $Z$ boson. The maximum cross section achieved reaches the value $3.4611 \times 10^{-4} \mathrm{pb}$ for $\tan \beta=5$ and $M 2=150$ $\mathrm{GeV}$ at a center of mass energy, $E_{C M}=560 \mathrm{GeV}$. A maximum of $3.0094 \times 10^{-4} \mathrm{pb}$ was also obtained for $\tan \beta=15$ and $M 2=150 \mathrm{GeV}$ at a center of mass energy, $E_{C M}=560 \mathrm{GeV}$ and again for the diagram no. 5. For $\tan \beta=35$ and $M 2=150 \mathrm{GeV}$ at a center of mass energy, $E_{C M}=580 \mathrm{GeV}$, the cross section obtained, reached the value $2.5145 \times$ $10^{-4} \mathrm{pb}$.

1. For $\tan \beta=5$ and $M 2=150, \sigma_{\max }=7.8383 \times 10^{-4} \mathrm{pb}$ at $E_{C M}=720 \mathrm{GeV}$.

2. For $\tan \beta=15$ and $M 2=150, \sigma_{\max }=5.2422 \times 10^{-4} \mathrm{pb}$ at $E_{C M}=820 \mathrm{GeV}$.

3. For $\tan \beta=35$ and $M 2=150, \sigma_{\max }=4.3095 \times 10^{-4} \mathrm{pb}$ at $E_{C M}=800 \mathrm{GeV}$.

4. For $\tan \beta=5$ and $M 2=300, \sigma_{\max }=3.3550 \times 10^{-4} \mathrm{pb}$ at $E_{C M}=1080 \mathrm{GeV}$.

5. For $\tan \beta=15$ and $M 2=300, \sigma_{\max } \mathrm{x}=8.8536 \times 10^{-5} \mathrm{pb}$ at $E_{C M}=960 \mathrm{GeV}$.

6. For $\tan \beta=35$ and $M 2=300, \sigma_{\max }=1.3291 \times 10^{-4} \mathrm{pb}$ at $E_{C M}=940 \mathrm{GeV}$.
The maximum cross section achieved reaches the value $1.6853 \times 10^{-5} \mathrm{pb}$ for $\tan \beta=5$ and $M 2=300$ $\mathrm{GeV}$ at a center of mass energy, $E_{C M}=880 \mathrm{GeV}$ for the diagram no. 2 which proceeds through the photon propagator, $\gamma$. A maximum of $1.0836 \times 10^{-4}$ pb was obtained for $\tan \beta=15$ and $M 2=300 \mathrm{GeV}$ at a center of mass energy, $E_{C M}=860 \mathrm{GeV}$ for the diagram no. 28 which proceeds through the exchange of the scalar neutrino propagator, $\tilde{v}_{e}$. For $\tan \beta=35$ and $M 2=300 \mathrm{GeV}$ at a center of mass energy, $E_{C M}=900 \mathrm{GeV}$, the cross section obtained, reached the value $1.8623 \times 10^{-4} \mathrm{pb}$ for diagram no. 28, total cross section achieved the following values: 
Table (1): Summary of the results obtained for the reaction, the reaction, $e^{-}\left(p_{1}\right) e^{+}\left(p_{2}\right) \rightarrow H^{-}\left(p_{3}\right) \widetilde{\chi}_{1}^{+}\left(p_{4}\right) \widetilde{\chi}_{1}^{o}\left(p_{5}\right)$ for $M 2=150 \mathrm{GeV}$

for $M 2=300 \mathrm{GeV}$

Table (2): Summary of the results obtained for $e^{-}\left(p_{1}\right) e^{+}\left(p_{2}\right) \rightarrow H^{-}\left(p_{3}\right) \widetilde{\chi}_{1}^{+}\left(p_{4}\right) \widetilde{\chi}_{1}^{o}\left(p_{5}\right)$

\begin{tabular}{|c|c|c|c|c|c|c|}
\hline \multirow{2}{*}{$\begin{array}{l}\text { Fig } \\
\text { no. }\end{array}$} & \multicolumn{2}{|c|}{$\sigma_{\tan \beta=5}$} & \multicolumn{2}{|c|}{$\sigma_{\tan \beta=15}$} & \multicolumn{2}{|c|}{$\sigma_{\tan \beta}=35$} \\
\hline & $E_{C M}$ & $\sigma(P b)$ & $E_{C M}$ & $\sigma(P b)$ & $E_{C M}$ & $\sigma(P b)$ \\
\hline 1 & 1040 & $1.578 \mathrm{e}^{-6}$ & 1140 & $1.591 \mathrm{e}^{-6}$ & 1160 & $1.642 \mathrm{e}^{-6}$ \\
\hline 2 & 900 & $2.029 \mathrm{e}^{-6}$ & 880 & $2.631 \mathrm{e}^{-6}$ & 920 & $2.793 \mathrm{e}^{-6}$ \\
\hline 3 & 1080 & $2.285 \mathrm{e}^{-7}$ & 1140 & $2.309 \mathrm{e}^{-7}$ & 1120 & $2.284 \mathrm{e}^{-7}$ \\
\hline 4 & 860 & $1.884 \mathrm{e}^{-6}$ & 840 & $2.498 \mathrm{e}^{-6}$ & 860 & $2.662 \mathrm{e}^{-6}$ \\
\hline 5 & 560 & $3.461 \mathrm{e}^{-4}$ & 560 & $3.009 \mathrm{e}^{-4}$ & 580 & $2.514 \mathrm{e}^{-4}$ \\
\hline 6 & 1000 & $5.321 \mathrm{e}^{-9}$ & 1060 & $7.959 \mathrm{e}^{-9}$ & 1060 & $8.589 \mathrm{e}^{-9}$ \\
\hline 7 & 1000 & $2.208 \mathrm{e}^{-10}$ & 1060 & $5.132 \mathrm{e}^{-10}$ & 1060 & $6.747 \mathrm{e}^{-10}$ \\
\hline 8 & 520 & $3.46 \mathrm{e}^{-4}$ & 560 & $6.914 \mathrm{e}^{-5}$ & 600 & $3.25 \mathrm{e}^{-5}$ \\
\hline 9 & 720 & $1.935 \mathrm{e}^{-5}$ & 720 & $3.21 \mathrm{e}^{-5}$ & 740 & $3167 e^{-5}$ \\
\hline 10 & 1480 & $8.854 \mathrm{e}^{-8}$ & 1500 & $6.575 \mathrm{e}^{-8}$ & 1480 & $5.211 \mathrm{e}^{-8}$ \\
\hline 11 & 1420 & $1.284 \mathrm{e}^{-6}$ & 1420 & $9.655 \mathrm{e}^{-7}$ & 1460 & $7.943 \mathrm{e}^{-7}$ \\
\hline 12 & 780 & $6.453 \mathrm{e}^{-7}$ & 880 & $2.07 \mathrm{e}^{-7}$ & 940 & $1.199 \mathrm{e}^{-7}$ \\
\hline 13 & 800 & $2.659 \mathrm{e}^{-5}$ & 800 & $2.125 \mathrm{e}^{-5}$ & 780 & $1.604 \mathrm{e}^{-5}$ \\
\hline 14 & 1160 & $1.000 \mathrm{e}^{-8}$ & 1160 & $9.325 \mathrm{e}^{-10}$ & 1160 & $1.491 \mathrm{e}^{-10}$ \\
\hline 15 & 1200 & $3.526 \mathrm{e}^{-9}$ & 1020 & $3.486 \mathrm{e}^{-9}$ & 1040 & $3.04 \mathrm{e}^{-9}$ \\
\hline 16 & 1080 & $7.656 \mathrm{e}^{-8}$ & 1040 & $6.839 \mathrm{e}^{-8}$ & 1040 & $5.885 e^{-8}$ \\
\hline 17 & 760 & $6.112 \mathrm{e}^{-7}$ & 800 & $1.673 \mathrm{e}^{-7}$ & 820 & $8.377 \mathrm{e}^{-8}$ \\
\hline 18 & 800 & $2.614 \mathrm{e}^{-5}$ & 780 & $2.092 e-5$ & 800 & $1.575 \mathrm{e}^{-5}$ \\
\hline 19 & 1180 & $1.320 \mathrm{e}^{-8}$ & 1060 & $3.037 \mathrm{e}^{-8}$ & 1040 & $4.102 \mathrm{e}^{-8}$ \\
\hline 20 & 1060 & $1.126 \mathrm{e}^{-7}$ & 1060 & $1.357 \mathrm{e}^{-7}$ & 1020 & $1.364 \mathrm{e}^{-7}$ \\
\hline 21 & 780 & $9.662 \mathrm{e}^{-7}$ & 800 & $4.052 \mathrm{e}^{-7}$ & 820 & $2.527 \mathrm{e}^{-7}$ \\
\hline 22 & 780 & $3.515 \mathrm{e}^{-5}$ & 760 & $4.295 \mathrm{e}^{-5}$ & 780 & $4.016 \mathrm{e}^{-5}$ \\
\hline 23 & 1440 & $3.303 e^{-7}$ & 1420 & $5.719 \mathrm{e}^{-7}$ & 1460 & $7.043 \mathrm{e}^{-7}$ \\
\hline 24 & 1440 & $1.885 \mathrm{e}^{-6}$ & 1420 & $1.913 \mathrm{e}^{-6}$ & 1460 & $1.844 \mathrm{e}^{-6}$ \\
\hline 25 & 780 & $1.023 \mathrm{e}^{-6}$ & 880 & $5.010 \mathrm{e}^{-7}$ & 960 & $3.607 \mathrm{e}^{-7}$ \\
\hline 26 & 820 & $3.58 \mathrm{e}^{-5}$ & 820 & $4.361 \mathrm{e}^{-5}$ & 820 & $4.087 e^{-5}$ \\
\hline 27 & 1120 & $4.403 \mathrm{e}^{-7}$ & 1040 & $7.352 \mathrm{e}^{-7}$ & 1080 & $8.531 \mathrm{e}^{-7}$ \\
\hline 28 & 800 & $3.304 \mathrm{e}^{-4}$ & 780 & $2.366 \mathrm{e}^{-4}$ & 800 & $1.849 \mathrm{e}^{-4}$ \\
\hline
\end{tabular}

\section{References}

1- T. Tsukamoto et. al., Phys. Rev. D 51 3153(1995).

2- J. L. Feng and M. J. Strassler, Phys. Rev. D 51 4661(1995).

3- J. L. Feng at. al., Phys. Rev. 52 -1418(1995).

4- M. Chen et. al., Phys. Reports 159 - 201(1988).

5- A. Salam, Rev. of Mod. Phys. 52 - 525(1980).

6- S. L. Glashow, Rev. of Mod. Phys. 52 -539(1980).

7- M. A. B. Beg and Sirlin, Phys. Reports 88 1(1980).

8- P. Fayet, Phys. Reports 105 -21(1984).

9- H. Georgi, Hadr. Jour. of Phys. 1 (1978) 155.

10- S. Bertolini, Nucl. Phys. B 272 (1986) 77.

\begin{tabular}{|c|c|c|c|c|c|}
\hline \multicolumn{2}{|c|}{$\sigma_{\tan \beta=5}$} & \multicolumn{2}{|c|}{$\sigma_{\tan \beta=15}$} & \multicolumn{2}{|c|}{$\sigma_{\tan \beta=35}$} \\
\hline$E_{C M}$ & $\sigma(P b)$ & $E_{C M}$ & $\sigma(P b)$ & $E_{C M}$ & $\sigma(P b)$ \\
\hline 1460 & $2.842 \mathrm{e}^{-6}$ & 1580 & $2.674 \mathrm{e}^{-6}$ & 1640 & $2.623 \mathrm{e}^{-6}$ \\
\hline 880 & $1.685 \mathrm{e}^{-5}$ & 880 & $2.229 \mathrm{e}^{-5}$ & 860 & $2.281 \mathrm{e}^{-5}$ \\
\hline 1560 & $4.106 \mathrm{e}^{-7}$ & 1560 & $3.856 \mathrm{e}^{-7}$ & 1560 & $3.785 \mathrm{e}^{-7}$ \\
\hline 860 & $8.531 e^{-6}$ & 860 & $1.130 \mathrm{e}^{-5}$ & 840 & $1.522 \mathrm{e}^{-5}$ \\
\hline 720 & $6.843 \mathrm{e}^{-6}$ & 720 & $4.632 \mathrm{e}^{-5}$ & 700 & $7.971 \mathrm{e}^{-5}$ \\
\hline 1280 & $2.079 \mathrm{e}^{-9}$ & 1300 & $2.293 \mathrm{e}^{-9}$ & 1360 & $2.25 \mathrm{e}^{-9}$ \\
\hline 1280 & $8.528 \mathrm{e}^{-10}$ & 1300 & $9.619 \mathrm{e}^{-10}$ & 1260 & $9.378 \mathrm{e}^{-10}$ \\
\hline 980 & $5.045 \mathrm{e}^{-7}$ & 980 & $4.638 \mathrm{e}^{-7}$ & 960 & $4.270 \mathrm{e}^{-7}$ \\
\hline 1060 & $2.082 \mathrm{e}^{-8}$ & 1100 & $2.771 \mathrm{e}^{-8}$ & 1120 & $2.789 \mathrm{e}^{-8}$ \\
\hline 1660 & $3.636 \mathrm{e}^{-7}$ & 1560 & $5.143 \mathrm{e}^{-7}$ & 1680 & $5.585 \mathrm{e}^{-7}$ \\
\hline 1520 & $1.681 \mathrm{e}^{-7}$ & 1460 & $1.506 \mathrm{e}^{-7}$ & 1540 & $1.402 \mathrm{e}^{-7}$ \\
\hline 1460 & $6.745 \mathrm{e}^{-9}$ & 1400 & $1.172 \mathrm{e}^{-8}$ & 1400 & $1.322 \mathrm{e}^{-8}$ \\
\hline 1220 & $4.413 \mathrm{e}^{-6}$ & 1280 & $5.285 \mathrm{e}^{-6}$ & 1280 & $5.393 e^{-6}$ \\
\hline 1260 & $5.885 e^{-9}$ & 1280 & $8.087 \mathrm{e}^{10}$ & 1280 & $1.541 \mathrm{e}^{-10}$ \\
\hline 1620 & $9.389 e^{-9}$ & 1220 & $1.634 \mathrm{e}^{-8}$ & 1240 & $2.087 \mathrm{e}^{-8}$ \\
\hline 1200 & $1.850 \mathrm{e}^{-8}$ & 1120 & $2.133 \mathrm{e}^{-8}$ & 1140 & $2.061 \mathrm{e}^{-8}$ \\
\hline 1180 & $1.142 \mathrm{e}^{-9}$ & 1120 & $2.566 \mathrm{e}^{-9}$ & 1100 & $3.071 \mathrm{e}^{-9}$ \\
\hline 1020 & $1.711 \mathrm{e}^{-6}$ & 1060 & $2.21 \mathrm{e}^{-6}$ & 1080 & $2.152 \mathrm{e}^{-6}$ \\
\hline 1640 & $3.025 \mathrm{e}^{-7}$ & 1240 & $4.192 \mathrm{e}^{-7}$ & 1200 & $5.023 \mathrm{e}^{-7}$ \\
\hline 1160 & $1.030 \mathrm{e}^{-8}$ & 1140 & $8.158 \mathrm{e}^{-9}$ & 1140 & $7.037 \mathrm{e}^{-9}$ \\
\hline 1160 & $1.289 \mathrm{e}^{-8}$ & 1120 & $2.578 \mathrm{e}^{-8}$ & 1160 & $2.991 \mathrm{e}^{-8}$ \\
\hline 1020 & $4.184 \mathrm{e}^{-7}$ & 1040 & $3.987 \mathrm{e}^{-7}$ & 1060 & $3.703 \mathrm{e}^{-7}$ \\
\hline 1600 & $1.170 \mathrm{e}^{-5}$ & 1660 & $1.319 \mathrm{e}^{-5}$ & 1640 & $1.344 \mathrm{e}^{-5}$ \\
\hline 1540 & $9.378 \mathrm{e}^{-8}$ & 1520 & $5.765 \mathrm{e}^{-8}$ & 1580 & $4.795 \mathrm{e}^{-8}$ \\
\hline 1480 & $7.612 e^{-8}$ & 1400 & $1.180 \mathrm{e}^{-7}$ & 1480 & $1.284 \mathrm{e}^{-7}$ \\
\hline 1300 & $1.078 \mathrm{e}^{-6}$ & 1300 & $9.946 \mathrm{e}^{-7}$ & 1320 & $9.304 \mathrm{e}^{-7}$ \\
\hline 1040 & $3.965 \mathrm{e}^{-6}$ & 1040 & $6.312 \mathrm{e}^{-6}$ & 1060 & $6.788 \mathrm{e}^{-6}$ \\
\hline 880 & $1.599 \mathrm{e}^{-5}$ & 860 & $1.084 \mathrm{e}^{-4}$ & 900 & $1.862 \mathrm{e}^{-4}$ \\
\hline
\end{tabular}

11- B.Y. Al-Negashi, T.A. El-Azim and I.E.A. AbdulMagead, I.R.E.PHY 259(2013) V7N3.

12- Ibrahim S. Mahmoud, T.A. El-Azim, I.R.E.PHY 488(2012) V6N6.

13- J. W. Williamson, Am. Jour. of Phys. 33 (1965)987.

14- J. F. Gunion and H. E. Haber, Phys. Rev. D 37 (1988) 2515.

15- I. J. Aitchison and A. J. Hey, Gauge Theories in Particle Physics, Adam Hilger Ltd., Bristol, 1983.

16- S. M. Bilenky and J. Hosek, Phys. Reports 90 (1982) 73.

17- S. Weinberg, Rev. of Mod. Phys. 52 (1980) 515.

18- M. F. Sohnius, Phys. Reports 128 (1985) 39. 
19- H. Baer et. al. Phys. Rev. D 48 (1993) R2978.

20- H. E. Haber, et. al., Nucl. Phys. B 161 (1979) 493.

21- A. Bartl et. al., Zeit. f. Phys. C 30 (1986) 441.

22- M. M. El-Kheishin, Phys. Rev. D 45 (1992) 4345.

23- H. E. Haber, Phys. Rev. D 54 (1996) 687.

24- E. Ma, Phys. Rev. D 49 (1994) 1663.

25- S. Okubo, Prog. of Theor. Phys. 27 (1962) 1949.

26- R. L. Pease, Inter. Jour. of Theor. Phys. 34 (1995) 183

27- F. Abe at. al., Fermilab Pub. 94/097-E CDF.
28- H.E. Haber and G. L. Kane, Phys. Rev. D 117 (1985) 75.

29- J. F. Gunion and H. E. Haber, Nucl. Phys. B 272 (1986) 1 ;

J. F. Gunion and H. E. Haber, Nucl. Phys. B 278 (1986) 449;

J. F. Gunion and H. E. Haber, Nucl. Phys. B 307(1988) 445.

30- H.E. Haber and D. Wyler, Nucl. Phys. B 323 (1989) 267.

31- J. Rosiek, Phys. Rev. D 41 (1990) 3464 